\title{
CORRELATION BETWEEN CENTRAL NERVOUS SYSTEM DAMAGE AND CLINICAL DISABILITY IN A SAMPLE OF EGYPTIAN MULTIPLE SCLEROSIS PATIENTS
}

\author{
By \\ Fathi Mahmoud Afifi Nasra', Mohamed Aly M. Abboud ${ }^{1}$, Mohamad
Hamed Rashad ${ }^{1}$, and Mohammad Aboulwafa Ahmad Abdullah ${ }^{1 *}$ \\ ${ }^{1}$ Neurology Department, Al-Azhar University, Cairo, Egypt \\ ${ }^{2}$ Diagnostic Radiology Department, Al-Azhar University, Cairo, Egypt \\ *Correspondence: wafamail@gmail.com
}

\begin{abstract}
Background: Multiple sclerosis (MS) is an autoimmune disease of the central nervous system that causes inflammatory as well as degenerative changes in the central nervous system

Objective: Studying the correlation between clinical disability of the disease and advanced volumetric MRI parameter in a sample of Egyptian MS patients

Patients and methods: Sixty MS patients and 63 age and sex matched healthy controls were recruited for the study. Clinical measures including expanded disability status scale (EDSS), 9-hole peg test (9HPT), 25Feet walk test (25FWT), and paced auditory serial addition test (PASAT) were used. 3D T1 MRI brain was acquired for measurement global as well as regional brain atrophy using FSL software, FLAIR sequence used for brain lesion load estimation; short tau inversion recovery (STIR) sequence was used for cervical spinal cord.

Results: Brain volume was significantly lower in MS patients compared to healthy controls, progressive phenotypes showed lowest values compared to clinically isolated syndromes and relapsing remitting MS. Brain Lesion load correlated with EDSS was high in early-MS subgroup, and no correlation to EDSS found at later-MS subgroup. High cervical lesion count was found in the study group and cervical lesions showed significant correlation with clinical disability. Thalamic atrophy was present in early-MS group compared to healthy controls, and thalamic volume correlated with clinical disability in this subgroup.

Conclusion: Egyptian patients showed relatively similar disease characteristics to western MS patients regarding clinical as well as radiological parameters. Heavier cord lesion burden, and strong correlation between lesion load and clinical disability early in the disease may account for the relatively more aggressive disease course in Egyptian population. Thalamic atrophy occurred early in MS, and may serve as a potential parameter in differentiating MS from other conditions.
\end{abstract}

Key words: Multiple Sclerosis, brain atrophy, cervical lesions, thalamic atrophy.

\section{INTRODUCTION}

Multiple sclerosis MS is an autoimmune demyelinating disease of the central nervous system that involves the brain and the spinal cord. Quantitative
MRI measures have shown to provide a better correlation to clinical disability measured by both global clinical scales as well as specific functional scales. Motor symptoms are relatively more common in African MS patients compared to other 
ethnicities (Langer-Gould et al., 2014, Filippi et al., 2016 and Hamdy et al., 2017).

Motor and spinal cord are by far the first presenting symptom in most patients in two large MS cohorts reaching 34\% compared to $20 \%$ for optic neuritis known to be the most common initial presentation in western cohorts (Zakaria et al., 2016).

MRI measures of disease activity is expressed through the number of active lesions (Gadolinium enhancing lesions and new/expanding T2 lesions), and recently brain atrophy rate has been introduced in clinical research as a measure of disease activity. A number of MRI studies assessed the influence of MS lesions on development of global and regional brain atrophy; however the influence tissue loss status on the correlation between lesion burden and clinical disability has not receive an adequate attention in MS research.

The application of lesion quantification as well as the volumetric assessment of brain and spinal cord has not been adequately assessed in multiple sclerosis patients in Egypt, and it is of interest adequately describe these measures in this particular group of patients given their different genetic and environmental profile.

The present study aimed to evaluate the clinical and quantitative radiological MRI features in an Egyptian population and to provide clear relationship between radiological and clinical measures of disability.

\section{PATIENTS AND METHODS}

Samples of MS patients from Egypt diagnosed according to McDonald's 2010 criteria were involved (Polman et al., 2011).

The sample represented the major clinical phenotypes including clinically isolated syndrome CIS. Another group of healthy controls were selected to be matched for both age and sex to compare the MRI atrophy measures between MS patients and healthy controls.

Assessment included demographic data for age, sex, occupation, marital status, and special habits of medical importance as well as disease duration, and the use of disease modifying drugs or other immunosuppressive drugs. A full standardized, clinical assessment of patients will be implemented with different clinical functional scales specific for MS.

\section{All patients had the following MRI sequences on 1.5 Philips Achieva MRI scanner:}

Brain: T2 and FLAIR sequences, for lesion load assessment; axial 3DT1 sequence, for atrophy assessment, Postcontrast T1-weighted sequence, for gadolinium-enhancing lesion quantification.

Cord: Sagittal T2-weighted/STIR sequence of the whole cord, for the assessment of the number of cord lesions. Cervical cord sagittal 3D T1-weighted sequence, for the assessment of cervical cord atrophy.

Lesion load quantification was assessed using semi-automated software commonly used in assessment of lesion 
volumes. Brain atrophy will be assessed using one of the validated methods of measurement of brain atrophy in multiple sclerosis that was based on lesion masking and segmentation techniques. Spinal cord atrophy was assessed by software that used semi-automated/fully automated assessment of the spinal cord.

\section{Ethical approval:}

The current investigation was executed based on the recommendations of the ethical committee, Faculty of Medicine, Al-Azhar University, Cairo, Egypt. All clinical interventions were illustrated obviously to all participants prior to study processing. Possible adverse events were exemplified, whereas, a written informed consent after a clear explanation of all study steps was obtained.

\section{Statistical Analysis:}

Comparison of lesion load, brain atrophy and spinal cord atrophy between different disease phenotypes as well as between patients and controls was assessed using independent sample t test. Analysis of variance was used to compare between different subgroups followed by post-hoc comparisons using t-test statistics. The correlation between MRI parameters and clinical disability measures was assessed using Spearman Rank Correlation coefficient. Assessment of the influence of tissue loss on the correlation between lesion load and clinical measures of disability was done using a Univariate Regression Analysis with brain and cord atrophy as covariates in the regression model.

\section{RESULTS}

The study has included 60 MS patients (41 females: 19 males) with mean age $32.6( \pm 10.5 \mathrm{SD})$, and 63 healthy controls
(40 females: 23 males), mean age 31.7 $( \pm 8.3 \mathrm{SD})$.

Main clinical and neuroimaging parameters were outlined in (table1).

Table (1): Main clinical and neuroimaging parameters of the study groups (Mean \pm SD)

\begin{tabular}{|c|c|c|c|}
\hline Study Groups & Controls & Patients & P values \\
\hline Parameter & $40: 23$ & $41: 19$ & 0.7 \\
\hline Sex (F:M) & $31.7321 \pm(8.3)$ & $32.5917 \pm(10.5)$ & 0.61 \\
\hline Age (years) & $0.291(0.03)$ & $0.273(0.02)$ & $<0.001$ \\
\hline Mean MSFC & $19.2(4.7)$ & $23.7(4.1)$ & $<0.001$ \\
\hline Mean 9HPT & $5.6(0.7)$ & $7.8(0.9)$ & $<0.001$ \\
\hline Mean 25FWT & $37.2(3.4)$ & $50.1(6.2)$ & $<0.001$ \\
\hline Mean PASAT & - & $2.6(1.8)$ & - \\
\hline EDSS & - & $4.9(4.4)$ & - \\
\hline $\begin{array}{c}\text { Disease Duration } \\
\text { (years) }\end{array}$ & - & $0.79(1.2)$ & - \\
\hline $\begin{array}{c}\text { Duration on DMT } \\
\text { (years) }\end{array}$ & & & \\
\hline
\end{tabular}


Lesion load in the patient study group (figure 1) correlated significantly with early-MS subgroup (disease duration of 5 years or less) compared to later-MS subgroup (Figure 2). Cervical cord lesions were present in $88 \%$ of patients, with $67 \%$ of patients have cervical lesions within 5 years of the disease course. Diffuse signal changes along the cord were observed in $67 \%$ of patients. Cord lesions correlated significantly with EDSS $(p=0.001$, $\mathrm{r}=0.530$ ), and correlated weakly with disease duration $(\mathrm{p}=0.04, \mathrm{r}=0.348)$, and no correlation found with brain lesions load.

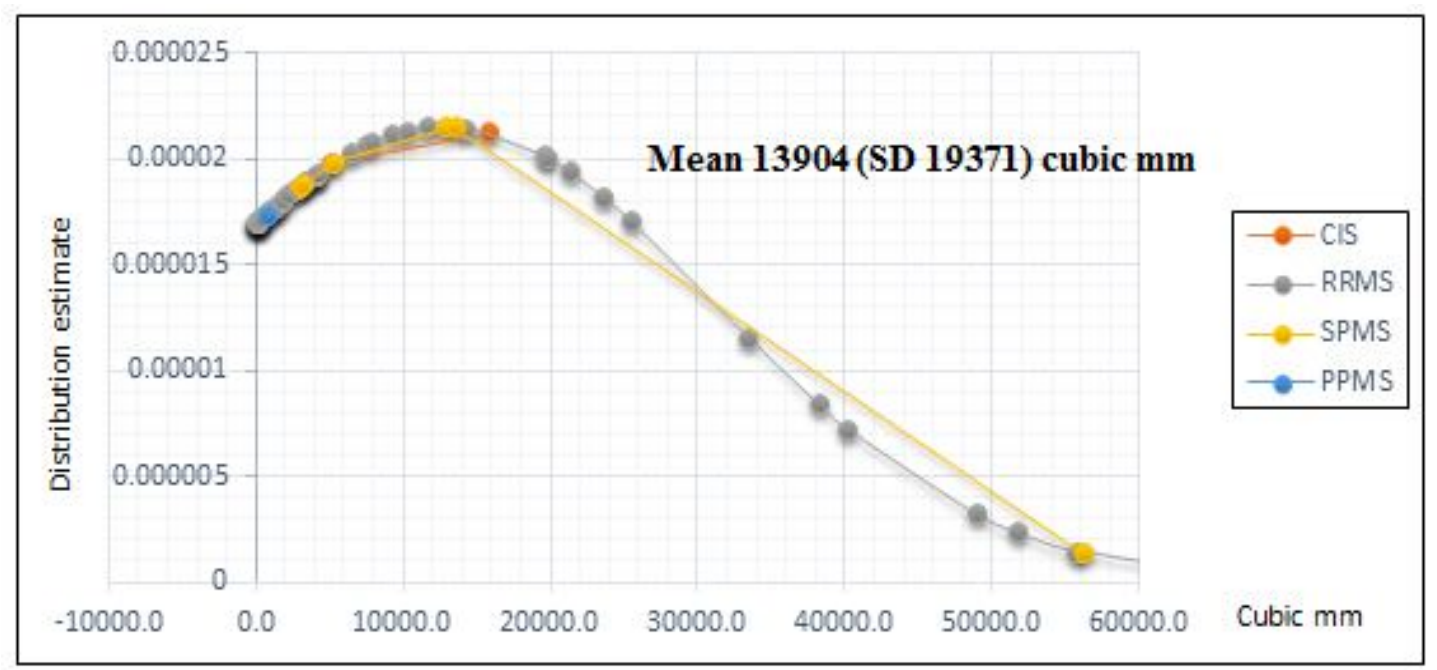

Figure (1): Mean lesion load distribution among MS patients with different phenotypes

CIS: Clinically Isolated Syndrome, RRMS: Relapsing Remitting Multiple Sclerosis, SPMS: Secondary Progressive Multiple Sclerosis, PPMS: Primary Progressive Multiple Sclerosis.

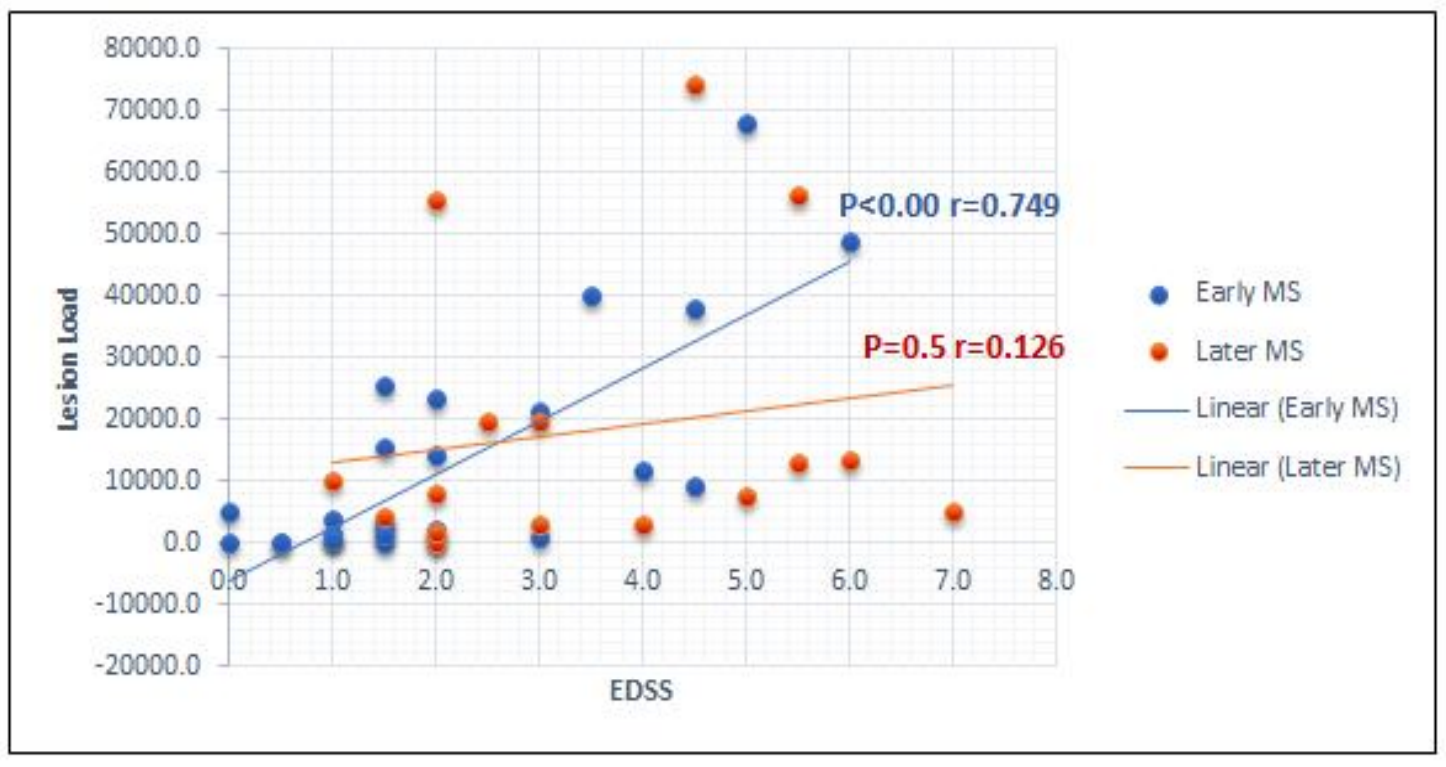

Figure (2): EDSS correlation with lesion load in early-MS and later-MS subgroups 
Main global brain volumetric between healthy controls and CIS patients parameters were significantly different were not significant $(p=0.12)$, in contrast between healthy controls and MS patients, to comparison between, CIS and RRMS and between different disease phenotypes $\quad(\mathrm{p}<0.01)$, CIS and SPMS $(\mathrm{p}<0.001)$ and using ANOVA. Post-hoc comparisons in $\quad$ RRMS and SPMS ( $\mathrm{p}<0.001)$ (Figure 3).

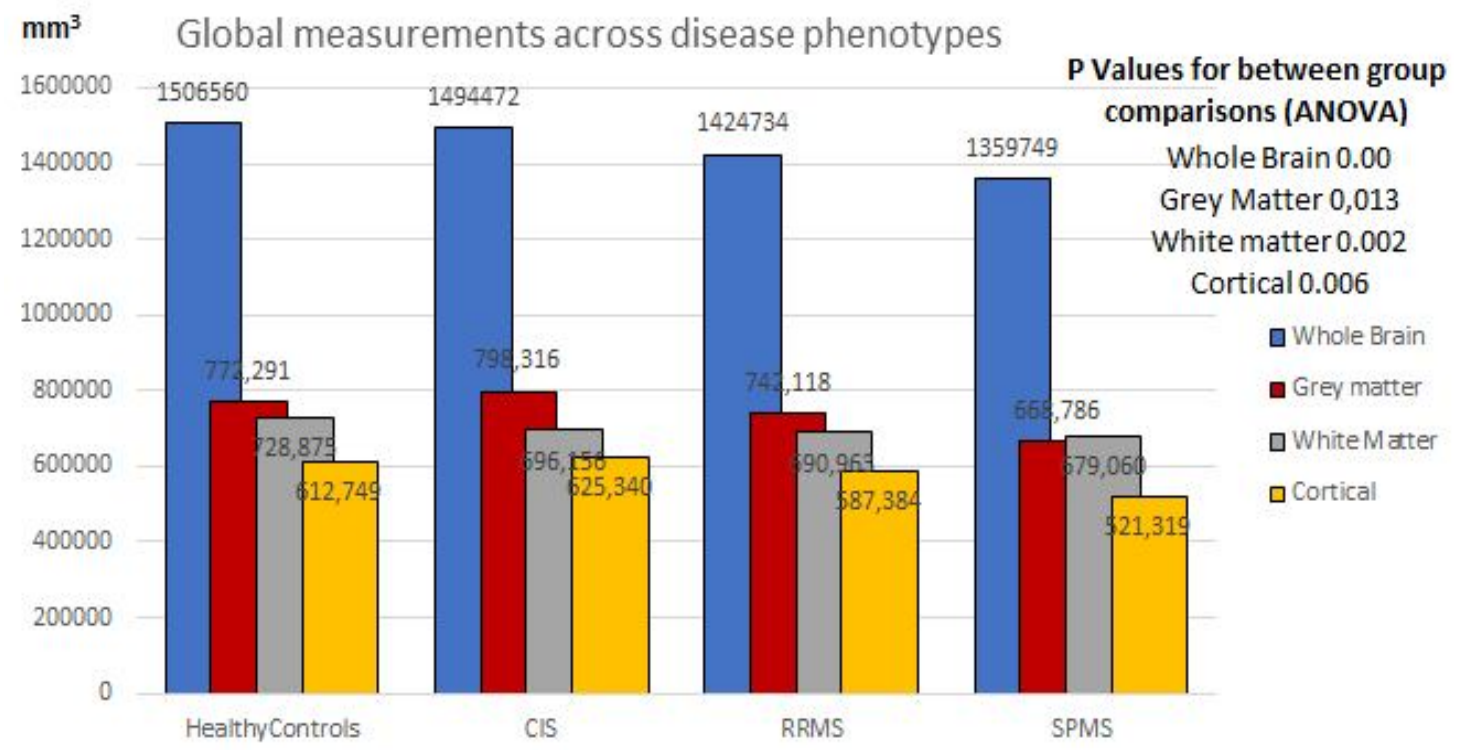

Figure (3): Mean whole Brain, Cortical volume, Grey matter volume, and white matter volume healthy controls and disease phenotypes

CIS: Clinically Isolated Syndrome, RRMS: Relapsing Remitting Multiple Sclerosis, SPMS: Secondary Progressive Multiple Sclerosis.

EDSS correlated moderately with global brain volume and cortical volume (Figure 4). Thalamic volume correlated significantly with EDSS only in early-MS subgroup, in contrast to later-MS subgroup (Figure 5). 


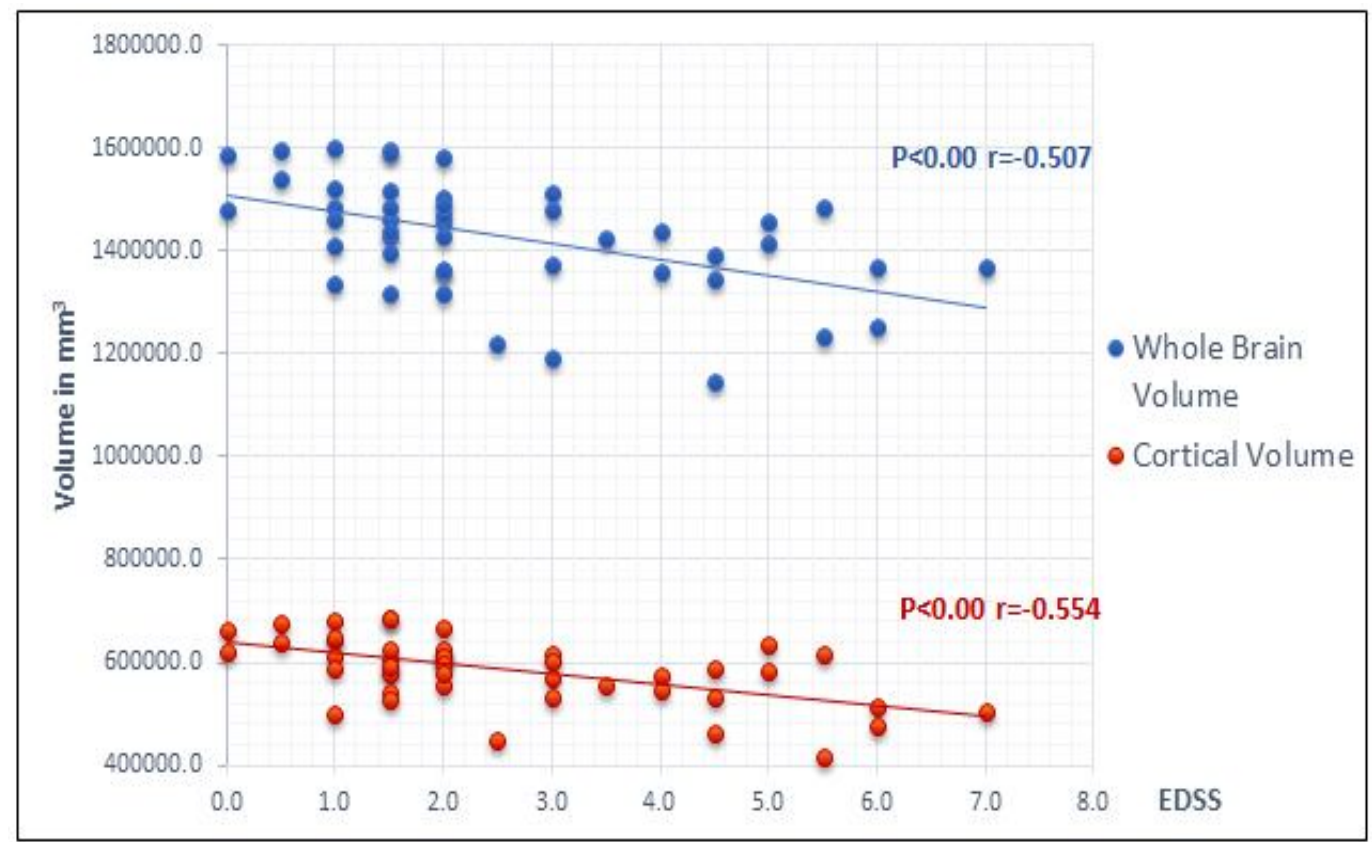

Figure (4): Correlation between EDSS score and whole brain volume and cortical volume

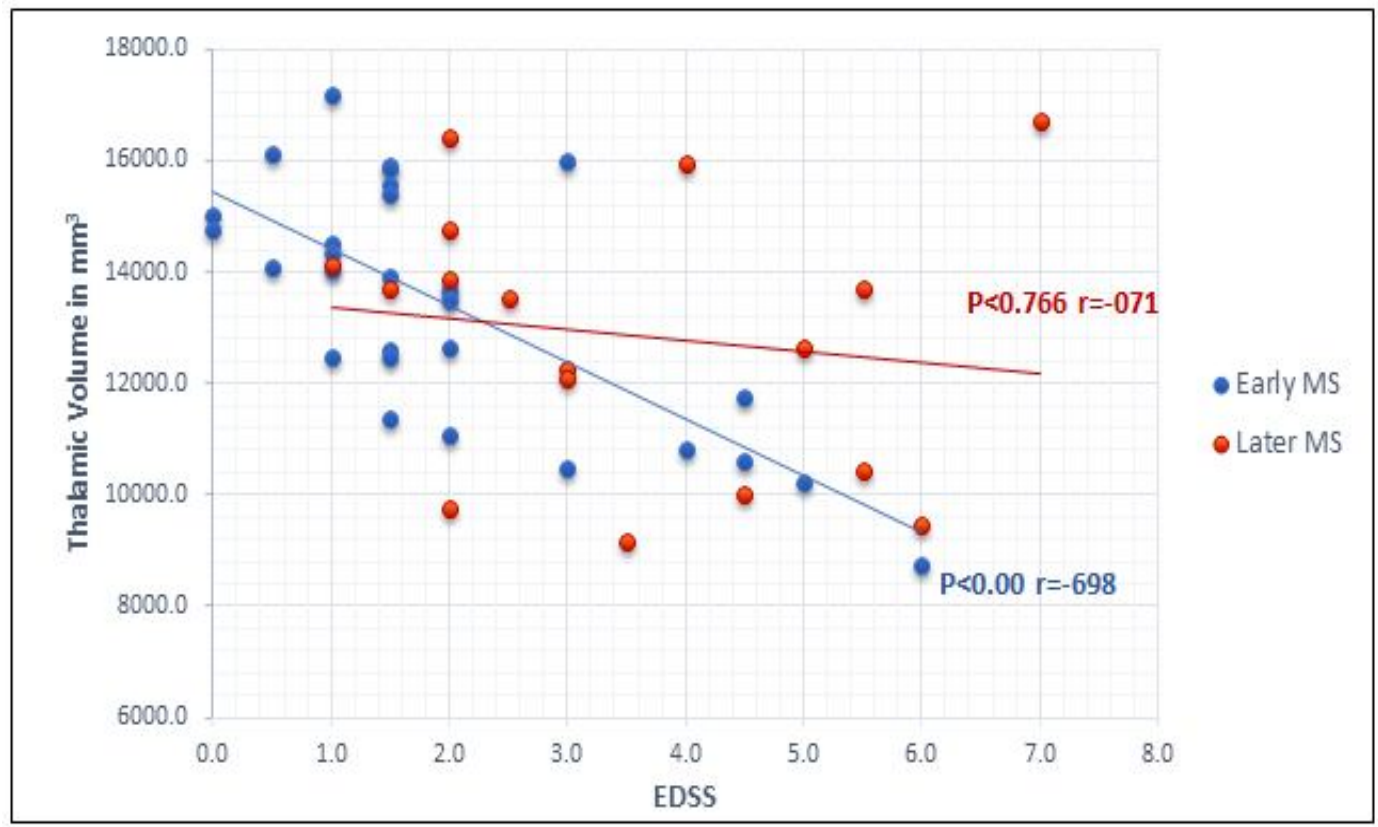

Figure (5): Correlation of EDSS and thalamic volume in early-MS and late-MS subgroups. 


\section{DISCUSSION}

In our sample of MS patients, the basic demographic features of the disease regarding age and sex were reflective of that expected from large national cohorts and otherwise international cohorts. Relapsing remitting MS was the main clinical phenotype $(78 \%)$ in our sample. However, primary progressive MS was only $2 \%$. This was reflective of the observed low prevalence of primary progressive phenotype in Egyptian cohorts compared to western cohorts (LangerGould et al., 2014, Zakaria et al., 2016 and Hamdy et al., 2017).

Genetic variance between relapsing forms and primary progressive MS has been demonstrated in a number of studies. However, correlation remains modest and only partially interprets the differences in clinical phenotypes (Kiselev et al., 2019).

Under-diagnosis of primary progressive MS may also interpret partly the low prevalence observed, due to similarities to other mimicking neurological conditions such as hereditary spastic paraparesis and leukodystrophies. The lack of access to specialized neurological practice in some regions in Egypt might also be a factor contributing to under-diagnosis (Hamdy et al., 2017).

In the current study, a moderate correlation between clinical disability scales and MRI lesion load was evident for the overall study sample. This correlation was highly significant in earlyMS subgroup (5 years or less of disease duration), while in those with more than 5 years of disease duration showed poor correlation. The level of correlation in the current study was relatively higher in comparison to Western studies. Early correlation with clinical disability with later loss of such correlation was well observed in other studies. The replication of the correlations pattern between EDSS and the brain lesion load between this study and western studies suggested no particular difference in the lesion nature that may interpret the apparent difference in disease aggressiveness (Brownlee et al., 2019).

Earlier stronger correlation indicated that lesions relatively resulted in clinically measurable effect by EDSS, which support the hypothesis that African/Egyptian MS have more frequent motoric presentation initially in the disease. Cervical cord lesion burden was present in most of the patient sample whether focal or diffuse cord abnormality. Cord lesions in established MS cases were frequent, and the prevalence depended upon the MRI sequences used and the stage of the disease. Early and frequent involvement of the cord is a known bad prognostic factor for MS disease course. In the current study, a relatively higher prevalence and better correlation to clinical disability early in disease course compared to available western data (Bot and Barkhof, 2009).

Genetic differences across different population may be one potential interpretation for differences between Egyptian and Western MS patients in focal cord involvement and its effect on clinical disability. Single study has demonstrated certain nucleotide polymorphism at the major histocompatibility complex region (HLADRB1*1501 allele) is associated with higher cervical cord lesion burden (Sombekke et al., 2009). 
In the current study, all global brain volume measurements were significantly lower in MS group compared to healthy controls. Also, all global brain volume measurements were significantly different between different MS phenotypes subgroups. However, cortical and grey matter volumes were the only significantly different between early and later disease duration subgroups. Those with CIS did not show any difference from healthy controls.

EDSS showed modest correlation to global brain volume, cortical and grey matter volumes but not the white matter volume. MSFC showed best correlation with cortical volume. PASAT test (the cognitive test in MSFC) showed correlation with all global brain volume measurements. Higher correlation was observed in patients with more than 5 years disease duration compared to early MS patients.

Several studies have shown that global brain volume at CIS or early relapsing MS show only modest correlation to clinical disability, and are less predictive of longterm clinical progression compared with focal MRI damage in MS (Sormani et al., 2017 and Brownlee et al., 2019).

This observation might have an appealing interpretation. Earlier disease course is expected to have less atrophic changes that take some time to develop, and inflammatory changes inside the brain result in increased water content inside the brain. This point is supported by the observation that CIS patients may have increased volume compared to controls as demonstrated by some studies. On the other hand, multiple active focal lesions are markers of more aggressive disease process and subsequently is expected to show stronger correlation to long-term disability in MS patients. However, with time course of the disease, -as demonstrated in the current studycorrelation with atrophy becomes stronger compared to focal lesion load (Brownlee et al., 2019).

Thalamic, caudate, putamen and hippocampal volumes were significantly lower in MS patients, with thalamic volumes being highly significantly lower. In early MS group, only thalamic volumes, hippocampal volumes, and left putamen volume clearly maintained that difference in comparison to healthy controls indicating that thalamic atrophy occurs early along the disease course. EDSS correlated with caudate volumes, and PASAT (cognitive component of MSFC) correlated with thalamic, putamen and caudate volumes.

These findings were similar to other studies that have demonstrated early changes in thalamic volumes observed in CIS and early relapsing MS, and predictive of conversion to clinically definite MS in CIS groups. It was difficult also to detect differences between CIS patients and early clinically definite MS in even studies with larger number of patients. However percent of thalamic atrophic changes over time were higher in CIS patients who subsequently developed clinically definite MS (Calabrese et al., 2011, Bergsland et al., 2012 and Zivadinov et al., 2013).

The mechanisms and reason behind early thalamic atrophy in MS remains to be elucidated. However, longitudinal MRI studies demonstrated that thalamic atrophy correlates with lesion burden 
early in relapsing phase of the disease, but later during the progressive course it is more correlated to microstructural damage rather than focal damage. This distinction was not observed in this study because of the smaller sample size, beside microstructural damage assessment was not implemented (Minagar et al., 2013 and Pontillo et al., 2019).

\section{CONCLUSION}

Egyptian patients showed relatively similar disease characteristics to western MS patients regarding clinical as well as radiological parameters. Heavier cord lesion burden, and strong correlation between lesion load and clinical disability early in the disease may account for the relatively more aggressive disease course in Egyptian population. Thalamic atrophy occurred early in MS and may serve as a potential parameter in differentiating MS from other conditions.

\section{REFERENCES}

1. Bergsland, N., Horakova, D., Dwyer, M. G., Dolezal, O., Seidl, Z. K., Vaneckova, M. and Zivadinov, R. (2012): Subcortical and cortical gray matter atrophy in a large sample of patients with clinically isolated syndrome and early relapsing-remitting multiple sclerosis. AJNR Am J Neuroradiol; 33(8): 1573-1578.

2. Brownlee, W. J., Altmann, D. R., Prados, F., Miszkiel, K. A., Eshaghi, A., Gandini Wheeler-Kingshott, C. A. M. and Ciccarelli, O. (2019): Early imaging predictors of long-term outcomes in relapseonset multiple sclerosis. Brain; 142(8): 22762287.

3. Calabrese, M., Rinaldi, F., Mattisi, I., Bernardi, V., Favaretto, A., Perini, P. and Gallo, P. (2011): The predictive value of gray matter atrophy in clinically isolated syndromes. Neurology; 77(3): 257-263.
4. Filippi, M., Rocca, M. A., Ciccarelli, O., De Stefano, N., Evangelou, N., Kappos, L. and Group, M. S. (2016): MRI criteria for the diagnosis of multiple sclerosis: MAGNIMS consensus guidelines. Lancet Neurol; 15(3): 292-303.

5. Hamdy, S. M., Abdel-Naseer, M., Shalaby, N. M., Elmazny, A. N., Nemr, A. A., Hassan, A. and Shehata, H. S. (2017): Characteristics and predictors of progression in an Egyptian multiple sclerosis cohort: a multicenter registry study. Neuropsychiatr Dis Treat; 13: 1895-1903.

6. Kiselev, I., Bashinskaya, V., Baulina, N., Kozin, M., Popova, E., Boyko, A. and Kulakova, O. (2019): Genetic differences between primary progressive and relapsingremitting multiple sclerosis: The impact of immune-related genes variability. Mult Scler Relat Disord; 29: 130-136.

7. Minagar, A., Barnett, M. H., Benedict, R. H., Pelletier, D., Pirko, I., Sahraian, M. A. and Zivadinov, R. (2013): The thalamus and multiple sclerosis: modern views on pathologic, imaging, and clinical aspects. Neurology; 80(2): 210-219.

8. Polman, C. H., Reingold, S. C., Banwell, B., Clanet, M., Cohen, J. A., Filippi, M. and Wolinsky, J. S. (2011): Diagnostic criteria for multiple sclerosis: 2010 revisions to the McDonald criteria. Ann Neurol; 69(2): 292-302.

9. Pontillo, G., Cocozza, S., Lanzillo, R., Russo, C., Stasi, M. D., Paolella, C. and Brunetti, A. (2019): Determinants of Deep Gray Matter Atrophy in Multiple Sclerosis: A Multimodal MRI Study. AJNR Am J Neuroradiol; 40(1): 99-106.

10. Sormani, M. P., Truffinet, P., Thangavelu, K., Rufi, P., Simonson, C. and De Stefano, N. (2017): Predicting long-term disability outcomes in patients with MS treated with teriflunomide in TEMSO. Neurol Neuroimmunol Neuroinflamm; 4(5): e379.

11. Zakaria, M., Zamzam, D. A., Abdel Hafeez, M. A., Swelam, M. S., Khater, S. S., Fahmy, M. F. and Gadallah, M. (2016): Clinical characteristics of patients with 
multiple sclerosis enrolled in a new registry in Egypt. Mult Scler Relat Disord: 10, 30-35.

12. Zivadinov, R., Havrdova, E., Bergsland, N., Tyblova, M., Hagemeier, J., Seidl, Z. and Horakova, D. (2013): Thalamic atrophy is associated with development of clinically definite multiple sclerosis. Radiology: 268(3), 831-841. 


\section{علاقة تلف الجهاز العصبي المركزي بالإعاقة الإكلينيكية لاىى عينة من مرضى التصلب المتعدد المصرين الإعين}

فتحي محمود عفيفي، محمد علي عبود*، محمد حامد رشاد، محمد أبوالوفا أحمد

قسمي الأعصاب والأثعة التثخيصية** ـ كلية الطب - جامعة الأزهر

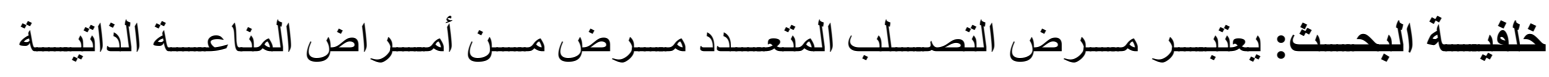

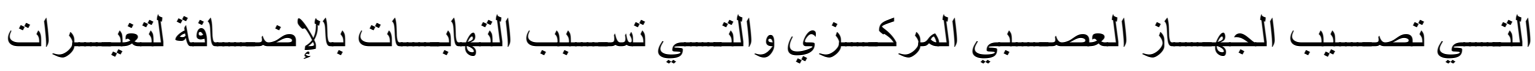
ضموريه بالجهاز العصبي المركزي

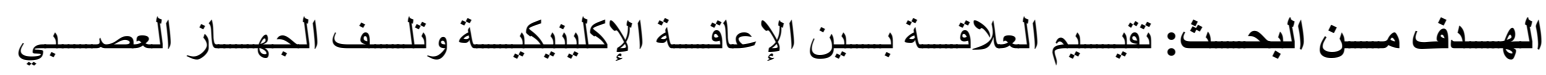

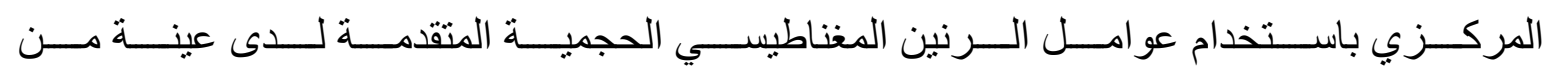

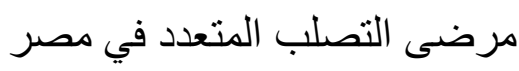

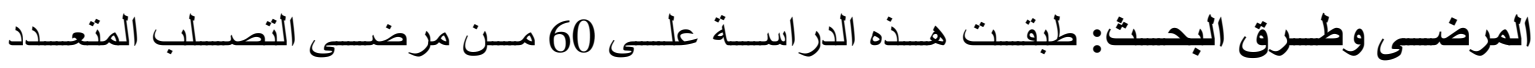

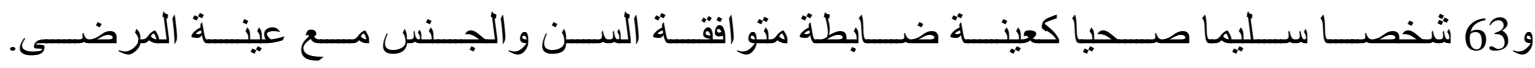

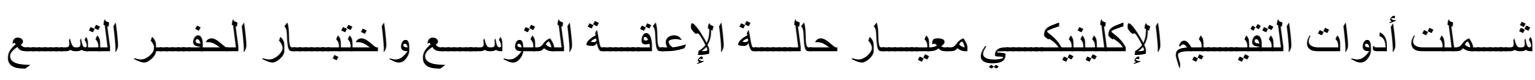

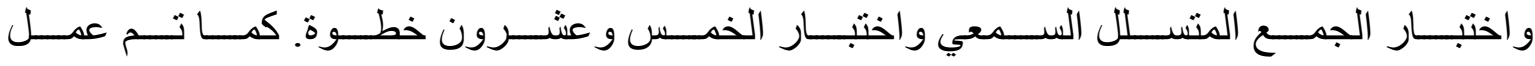

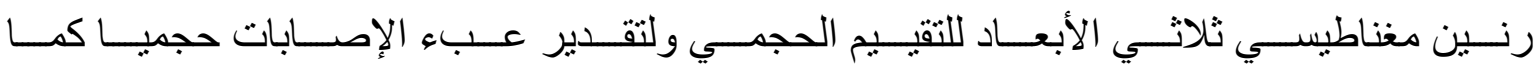
تم عمل فحص ستير لتقييم إصابات الحبل الثوكي.

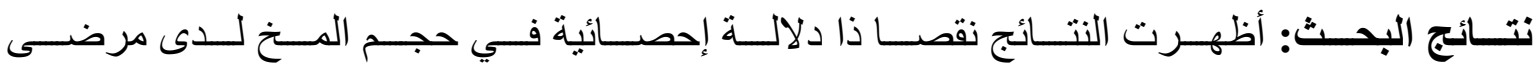

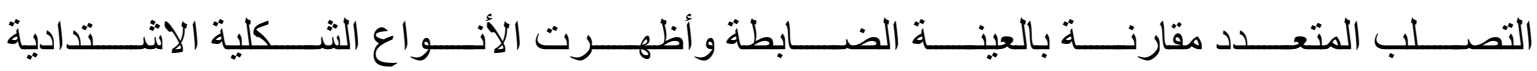

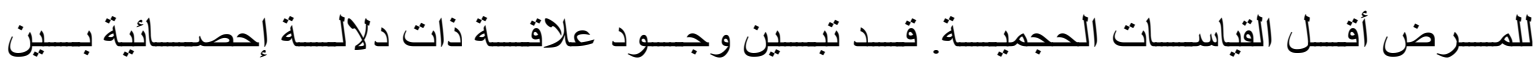

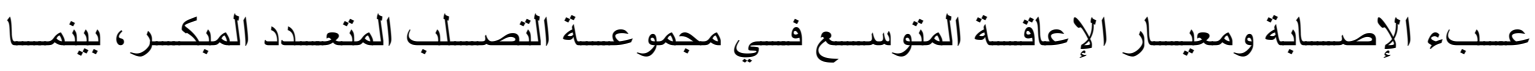

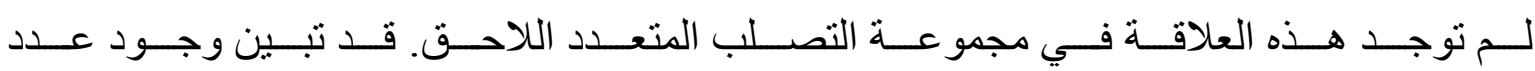

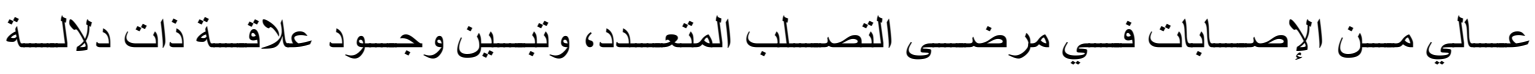

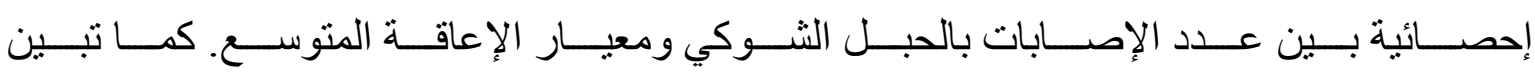

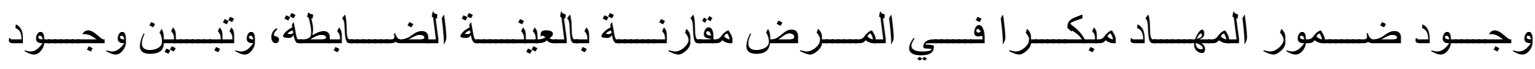
علاقة بينها وبين الإعاقة الإكلينيكية. 
الاســتتـاج: تتشــابه خصـــائص مــرض التصــلب المتعــدد فــي مصــر مــع التصـــلب المتعــدد

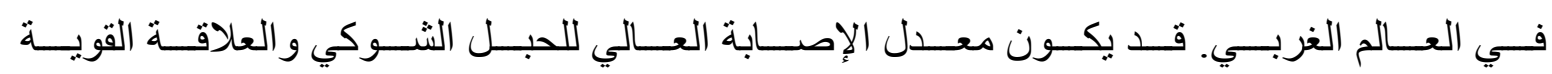

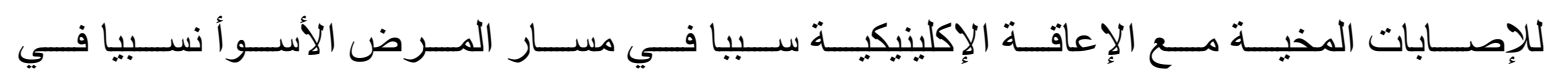

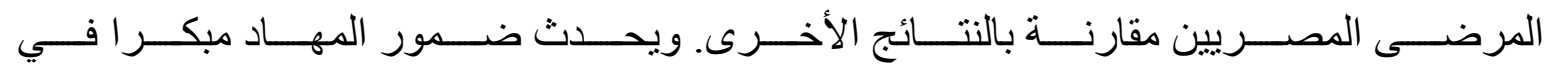

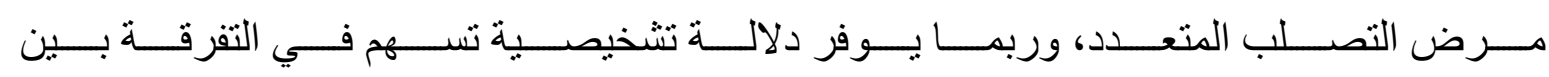
التصلب المتعدد و الأمر اض الأخرى. 